\title{
Transient extreme ultraviolet measurement of element-specific charge transfer dynamics in multiple- material junctions
}

Jonathan M. Michelsen, William T. Denman, Scott K. Cushing

Jonathan M. Michelsen, William T. Denman, Scott K. Cushing, "Transient extreme ultraviolet measurement of element-specific charge transfer dynamics in multiple-material junctions," Proc. SPIE 10926, Quantum Sensing and Nano Electronics and Photonics XVI, 109262A (1 February 2019); doi: $10.1117 / 12.2507473$ 


\title{
Transient Extreme Ultraviolet Measurement of Element-Specific Charge Transfer Dynamics in Multiple-Material Junctions
}

\author{
Jonathan M. Michelsen ${ }^{\text {a }}$, William T. Denman ${ }^{\mathrm{a}}$, Scott K. Cushing*a \\ ${ }^{a}$ Division of Chemistry and Chemical Engineering, Noyes Laboratory of Chemical Physics, \\ California Institute of Technology, Pasadena, California 91125
}

\begin{abstract}
The absorption of solid state materials in complex photonic and optoelectronic devices overlap in the visible spectrum. Due to the overlap of spectral features, ultrafast measurements of charge carrier dynamics and transport is obscured. Here, the element specificity of transient extreme ultraviolet (XUV) spectroscopy is advanced as a probe for studying photoexcited charge transport in multiple-material junctions. The core-hole excited by the XUV transitions also imparts structural information on to the probed electronic transition. Transient XUV can therefore measure electron and averaged phonon dynamics for each elemental species in a junction. Application to polaron measurement in $\alpha-\mathrm{Fe}_{2} \mathrm{O}_{3}$, valley-specific scattering in $\mathrm{Si}$, and charge transfer in a nanoscale $\mathrm{Ni}^{-\mathrm{TiO}_{2}}$-Si junction will be discussed.
\end{abstract}

Keywords: transient extreme ultraviolet spectroscopy, time-resolved energy transfer, element-specific carrier and phonon dynamics, transient absorption, transient reflectivity, nanoelectronics, photonic material

\section{INTRODUCTION}

Carrier and phonon scattering dynamics dictate the charge transfer within and between materials used for optoelectronic and electronic applications. In a nanoscale device, charge and heat transport occur on the same time and length scale as these scattering processes ${ }^{1-3}$. The assumption that charge dynamics can be separated in to bulk and interfacial components is no longer necessarily true in device-averaged measurements. For single materials and simple junctions, optical pump-probe and multidimensional spectroscopies have made significant progress towards understanding dynamics of the photoexcited state $e^{4-6}$. However, these techniques encounter difficulties when probing multiple-material junctions with overlapping absorption spectra in the region of interest. Unfortunately, the transition metal oxides, semiconductors, and metals that are combined for optoelectronic devices generally possess overlapping electronic transitions in the visible to infrared region.

Recent advances in high harmonic generation have allowed the production of attosecond and longer XUV pulses in table-top instrumentation ${ }^{7-11}$. In the extreme ultraviolet (XUV) region, a photoexcited core to valence level transition is measured. The energetic spacing between each element's transition energy, even in the XUV region, allows for element-specific probing. Visible light pump and element-specific XUV probe experiments are therefore possible. Further, with appropriate analysis of the core-hole perturbation, the spectral signatures of photoexcited electron and holes can be energetically separated in the transient XUV spectrum ${ }^{12-14}$. The averaged lattice dynamics due to excited phonon modes can also be measured ${ }^{15,16}$.

In this paper, the application of transient XUV to solid state systems and junctions will be discussed in terms of measuring carrier and lattice dynamics. This discussion includes the difference in core-hole screening for these materials and the appropriate theoretical framework to understand their excited state dynamics. The simultaneous observation of carriers and vibrational dynamics was first achieved in the gas phase ${ }^{11}$. The additional many-body effects present in a solid required further theoretical development to interpret the XUV spectral changes ${ }^{15}$. The evolution of measuring transition metal oxides, to covalently bonded semiconductors, to metals is described. This evolution culminates in the presented element specific measurement of hole transfer in a photoexcited $\mathrm{Ni}^{-} \mathrm{TiO}_{2}$ - $\mathrm{Si}$ junction. In addition to understanding charge and heat transfer in nanoelectronic and nanophotonic junctions, transient XUV spectroscopy also has the potential to guide understanding of dynamics in correlated quantum materials, such as ferroic materials, and superconductors $^{17}$. 


\section{APPLICATION OF TRANSIENT XUV TO SOLID STATE MATERIALS}

Transient XUV spectroscopy has been applied to resolve complex dynamics in solid state systems. This includes carrier dynamics in metal oxides ${ }^{18-21}$, polarons ${ }^{16,22,23}$, multilayer junctions ${ }^{24}$, as well as carrier-phonon and phonon-phonon scattering in semiconductors ${ }^{13-15,25}$.

\subsection{Transition metal oxides: Charge transfer and small polarons}

In a transition metal oxide, the localized $3 \mathrm{~d}$ valence levels interact strongly with the core-hole perturbation. The resultant XUV spectrum is dominated by atomic multiplet splitting, which energetically shifts, splits, and renormalizes the $3 \mathrm{~d}$ transitions. While the atomic multiplet splitting and charge-transfer interactions have so far prevented the extraction of carrier energies, the interactions themselves act as a sensitive probe of charge state and local lattice deformations in these often polar materials ${ }^{18-21}$.
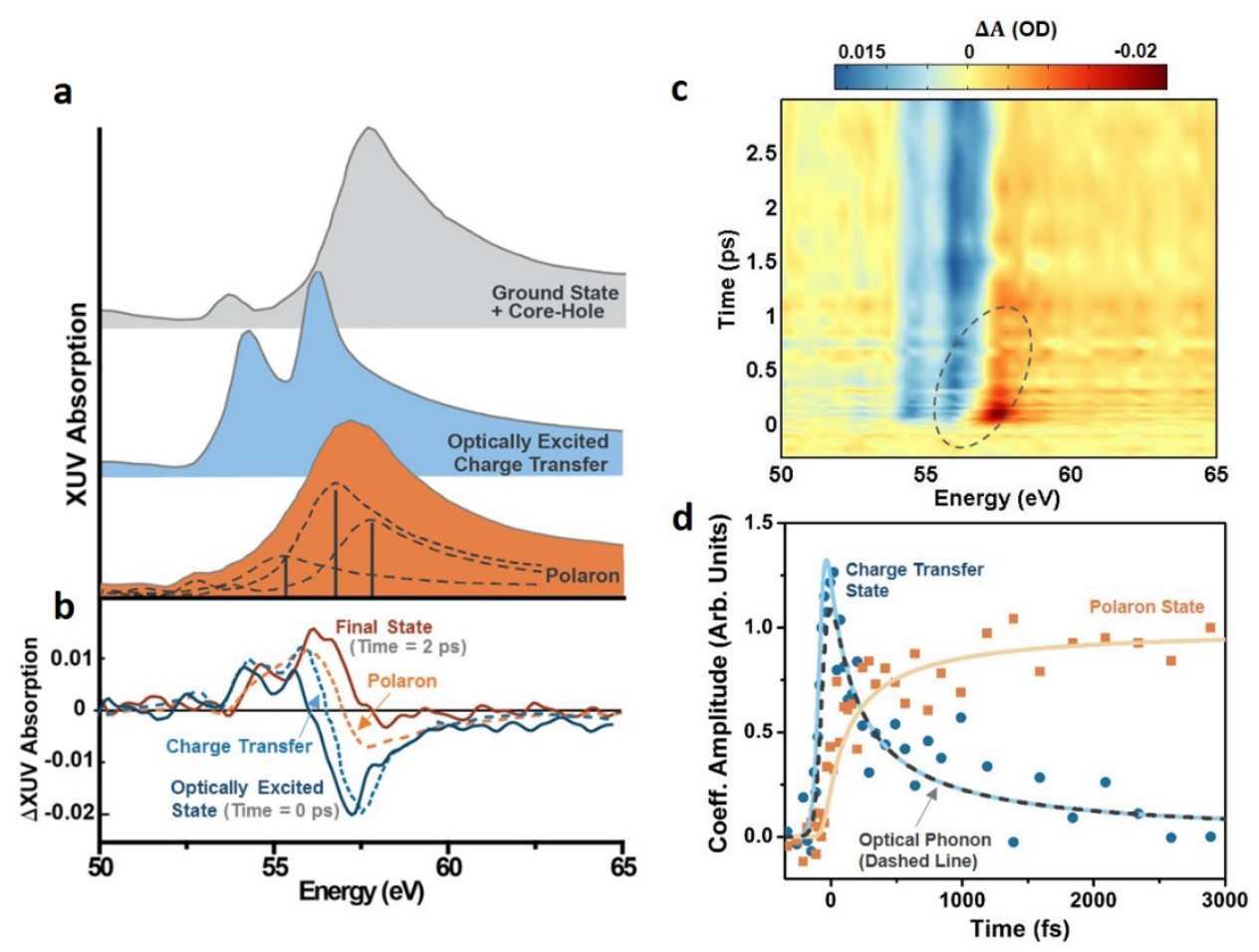

Figure 1. a The predicted ground state, photoexcited O $2 p$ to Fe $3 d$ ligand to metal charge transfer state, and the small polaron XUV absorption. b The differential absorption (solid lines) measured in the experiment is compared to the difference in the ground and excited or ground and polarn state spectrum from part a (dashed lines). The experimental differential absorption lineouts are well described by the ligand to metal charge transfer and a final small polaron state. $\mathbf{c}$ The full differential absorption for the experimental lineouts in part b. The differential transient absorption spectrum shows features of increased (blue) and decreased (red) absorption near 55 to $56 \mathrm{eV}$. Highlighted by the circle, the zero-crossing energy displays a blueshift in the first $500 \mathrm{fs}$. d The experimental TA at times of 0 ps and 2 ps after the pump pulse are used to extract the amplitude of the charge-transfer and small polaron states (data markers). A corresponding rate equation model fit is displayed as solid lines. Shown by the dashed line, the rate equation fit for the effective phonon population is estimated based on the electron population. Reprinted with permission from Ref. 16. Copyright 2017 Macmillan Publishers Limited, part of Springer Nature.

For example, small polarons are responsible for the ultrafast trapping process and decreased mobility measured in hematite $\left(\alpha-\mathrm{Fe}_{2} \mathrm{O}_{3}\right)$. Their formation limits the bulk transport and recombination in high efficiency hematite photoelectrodes; the application of hematite for improved solar water splitting is impeded. Transient XUV spectroscopy of the $\mathrm{Fe} \mathrm{M}_{2,3}$ edge was used to measure the change in atomic multiplet splitting following the photoexcited ligand-tometal charge transfer (LMCT) from the $\mathrm{O} 2 \mathrm{p}$ to the $\mathrm{Fe} 3 \mathrm{~d}$ orbitals (Figure 1a) ${ }^{16}$. Transitions between $\mathrm{d}$ states were ruled out by the measured transient signal (Figure 1b) ${ }^{21}$. After photoexcitation, the oxygen should stabilize the excess iron electron density by a change in lattice constant, resulting in the formation of small polarons. Specifically, the small polaron is predicted to lead to a threefold energy splitting of the $3 p$ core levels (Figure 1a). This prediction matches the 
measured transient XUV signal after 2-3 ps (Figure 1c). By fitting the results to a rate equation, the small polaron state formation is quantified to occur in less than $100 \mathrm{fs}$, or during the first optical-phonon scattering events (Figure 1d). The small polaron formation then continues for 2-3 ps and persists until the photoexcited carriers decay. The small polaron formation is also measured to be dependent on excitation wavelength. The formation rate was found to be the highest near the band gap. As excitation wavelength increases, the non-thermal phonon bath temperature increases. The rise in the lattice energy leads to increased hopping rate of the polaron, as well as increased hopping radius of localized carries, prolonging recombination and giving an increased excited-state mobility.

More recently, hematite's surface electron dynamics were characterized using transient XUV in a reflectivity geometry $^{22}$. It was concluded that surface defects and grain boundaries have a negligible impact on carrier localization. Instead, small polaron formation was measured to cause lattice expansion on the time scale of surface trapping, consistent with the results of Figure 1. XUV transient absorption was also used to study goethite $(\alpha-\mathrm{FeOOH})$ nanorods to determine how local field effects and geometry modify the polaron formation ${ }^{23}$. Like hematite, goethite features a $\mathrm{Fe}^{3+}$ center with octahedrally coordinated oxygen ligands. In goethite, iron atoms occupy $1 / 2$ of interstitial spaces, compared to $2 / 3$ for hematite. As a result, the iron-iron bond lengths in goethite are $5 \%$ longer when compared with those in hematite. Using transient XUV spectroscopy, the goethite small polaron formation time increases with energy from $70 \pm$ $10 \mathrm{fs}$ at $2.2 \mathrm{eV}$ to $359 \pm 30 \mathrm{fs}$ at $2.6 \mathrm{eV}$. Even when measurements were repeated with varying excitation wavelength, the average polaron formation time for goethite $(80 \pm 30 \mathrm{fs})$ is measured to be significantly lower than for hematite $(90 \pm 5$ fs).

\subsection{Group IV semiconductors: Carrier and lattice dynamics}

Covalently bonded semiconductors with delocalized valence orbitals, namely $\mathrm{Si}$ and $\mathrm{Ge}$, can screen the corehole perturbation sufficiently that the measured XUV spectra is close in form to the unoccupied density of states. This allows for extraction of the electron and hole energies from the transient XUV spectrum after photoexcitation. The XUV probe measures the sum over dipole allowed transitions in k-space. Therefore, the electron and hole energies are only measured in non-degenerate valleys. The average lattice dynamics are also measured through the core-hole's sensitivity to local structural changes.
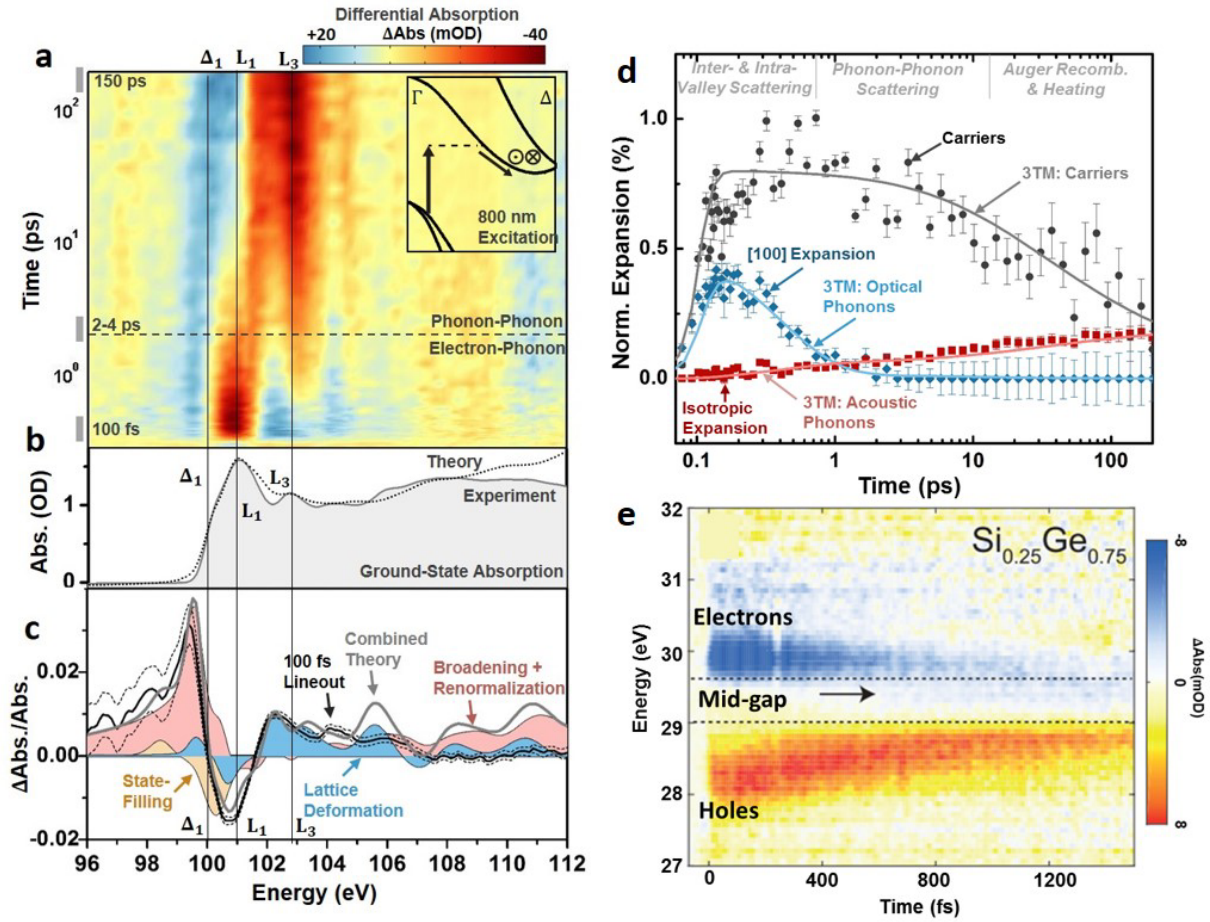

Figure 2. a The differential XUV absorption of the $\mathrm{Si}_{2,3}$ edge following $800 \mathrm{~nm}$ excitation. The inset displays some of the possible excitation and scattering pathways for valence electrons. b The measured ground state XUV absorption (grey area) 
compared to the theoretically predicted absorption (dotted line). The three critical points of the XUV absorption and the corresponding symmetry points in the Brillouin zone are marked. c Theoretical components of the excited state differential XUV signal at $100 \mathrm{fs}$ after photoexcitation. The experimental data (black solid line; the dashed lines represent the 95\% confidence intervals) is plotted versus the combined theoretical prediction (solid grey line) of the state-filling, lattice deformation, and broadening or renormalization components (colored areas). d The extracted electron, optical, and acoustic phonon amplitudes versus time match the known Auger, carrier-phonon, and phonon-phonon scattering times for $\mathrm{Si}$, represented here in a three-temperature model (3TM). e The theoretically processed differential XUV absorption for a $\mathrm{Si}: \mathrm{Ge}$ alloy measures the energy of electrons and holes as a function of time after photoexcitation. Note the filling of mid-gap states. Figures a-d and e are reproduced from References 15 and 14, respectively, under the Creative Commons Attribution 3.0 license.

Although the core-hole effects are reduced in Group IV semiconductors, they cannot be neglected completely. In $\mathrm{Si}$, for example, the electron and hole dynamics are still partially masked by the core-hole perturbation (Figure 2$)^{15}$. In Figure 2a, valence electrons are photoexcited across the bandgap into the $\Delta$ valley $(800 \mathrm{~nm}, 1.55 \mathrm{eV})$ via an indirect optical transition. The $\mathrm{Si} 2 \mathrm{p} \mathrm{L}_{2,3}$ edge $(100 \mathrm{eV})$ is measured during the carrier thermalization and recombination process. The resultant change in the XUV spectrum cannot be described purely by the increased and decreased absorption expected from photoexcited electrons and holes, as the pump only gives $1.55 \mathrm{eV}$ of energy to the valence electrons. The measured differential absorption spectrum would otherwise imply the creation of holes at energies up to $10 \mathrm{eV}$ above the valence band edge. Instead, the transition density of states must be corrected for the core hole perturbation using a BetheSalpeter calculation (Figure 2b). Similar to the metal oxides, both electronic and structural dynamics are predicted to be present in the measured transient XUV spectrum (Figure 2c).

The Si transient XUV absorption spectrum is modelled for ground and photoexcited states as follows ${ }^{15}$. First, the ground state is predicted using a single plasmon pole model and the Bethe-Salpeter equation (BSE) with density functional theory (DFT). Then, the excited state XUV spectrum is fit by approximating the contributions of excited state broadening, renormalization, state-filling, and structural dynamics. The structural dynamics are included by using equilibrium distortions and expansions of the ground state Si unit cell. Despite these approximations, the carrier and phonon dynamics quantified from such a fit agree with the known scattering constants of Si (Figure 2d). In particular, the scattering times for electron-optical phonon scattering, electron-acoustic phonon scattering, and optical acoustic phonon scattering were fit as $30 \mathrm{fs}, 500 \mathrm{fs}$, and $400 \mathrm{fs}$, respectively. The Auger recombination time matches the Richter model ${ }^{26}$.

The carrier and phonon dynamics have also been measured in optically excited Ge nanocrystalline thin films using the $\mathrm{Ge}_{4,5}$ edge $(30 \mathrm{eV})^{13}$. In this case, the photoexcited XUV spectrum is modelled by separating the contributions of electronic state blocking and band shifts; an approximation of the full Bethe-Salpeter equation calculations. The electron and hole energies are extracted as a function of time (Figure 2d). For Ge alone, the photoexcited carrier decay indicates a Shockley-Read-Hall first-order recombination mechanism. For a $\mathrm{Si}_{0.25} \mathrm{Ge}_{0.75}$ alloy, the differential XUV spectrum possesses a midgap feature that corresponds to trap states filling near the conduction band $^{14}$. XUV transient reflectivity, which provides access to a larger range of samples than a transmission measurement, can also be used to extract the dielectric function of crystalline Ge after photoexcitation ${ }^{25}$. Such transient reflectivity measurements have been used to directly measure the complex dielectric function in crystalline $\mathrm{Ge}, \alpha-\mathrm{Fe}_{2} \mathrm{O}_{3}$, and a $\mathrm{Fe}_{2} \mathrm{O}_{3} / \mathrm{NiO}$ heterojunction ${ }^{24}$.

\subsection{Metals: Change in Fermi level}

In metals, the electron wavefunctions are highly delocalized and mobile. Based on the trends from metal-oxides and Group IV semiconductors, the XUV absorption is expected to directly correlate to the unoccupied density of states. However, the core-hole pertubation creates a large number of states near the Fermi level ${ }^{27}$. This many body effect, often referred to as the orthogonality catastrophe or a white-line, distorts the final transition density of states. The core-hole can be treated perturbatively as a localized scattering impurity in the metal. The core-hole perturbation is then found to create an exponentially increasing number of possible electron configurations when the XUV energy approaches the fermi level. The x-ray absorption spectrum of a metal therefore have a sharp rise at the edge onset, the white line, followed by a power law tail at higher energies. This spectrum is analytically calculable by many-body theory ${ }^{27}$, accurately predicting ground-state XUV absorption for a metal using the Fermi level, temperature, and a phase factor that depends on the Fermi level energy, temperature, and band structure. Using this model, the measured transient XUV spectrum can be decribed as changes in the Fermi level energy and temperature after photoexcitation. The exact electron 
and hole distributions is obscured. The metallic core-hole interactions have also been discussed in terms of Fano-type configuration interaction where the core to d-level excitation interferes with the continuum of d-electron excitations ${ }^{28}$.

\subsection{Charge transport dynamics in multiple-material junctions}

Sections 2.1-2.3 outlined that transient XUV spectroscopy can measure element-specific electronic and structural dynamics following photoexcitation. This allows, for example, the charge and heat transport between the different layers of a metal-oxide-semiconductor junction to be quantified. The transient response of a $\mathrm{Ni}^{-} \mathrm{TiO}_{2}-\mathrm{Si}$ junction (Figure 3a) following photoexcitation of Si with $800 \mathrm{~nm}$ light is shown in Figure 3b. Using a broadband XUV probing pulse, the $\mathrm{Ti} \mathrm{M}_{2,3}(32.6 \mathrm{eV})$, $\mathrm{Ni} \mathrm{M}_{2,3}(66,68 \mathrm{eV})$, and $\mathrm{Si}_{2,3}(100 \mathrm{eV})$ edges are measured from 50 fs to $200 \mathrm{ps}$ after photoexcitation. Approximately $50 \mathrm{fs}$ after photoexcitation, there is an increase in the Ni Fermi level but no change in the $\mathrm{Ti}$ edge, indicating tunneling of holes from $\mathrm{Si}$ through the $\mathrm{TiO}_{2}$ layer to $\mathrm{Ni}$ (Figure 3c). The tunneling of photoexcited holes from Si to Ni could originate in the built-in field of the p-doped junction as well as the optical field of the laser pulse. On the picosecond time scale, the hole population on the $\mathrm{Ni}$ is measured to decrease, while the change in the charge state of the $\mathrm{TiO}_{2}$ indicates the acceptance of holes (Figure 3d). The back-diffusing holes in the $\mathrm{TiO}_{2}$ lead to recombination of the photoexcited electron population in $\mathrm{Si}$ on a tens of picoseconds timescale. The hole population in the $\mathrm{TiO}_{2}$ is measured to be depleted by the electron-hole recombination on a hundreds of picosecond timescale. The heat transfer between the three layers is also measured in the transient XUV spectra at this time scale.
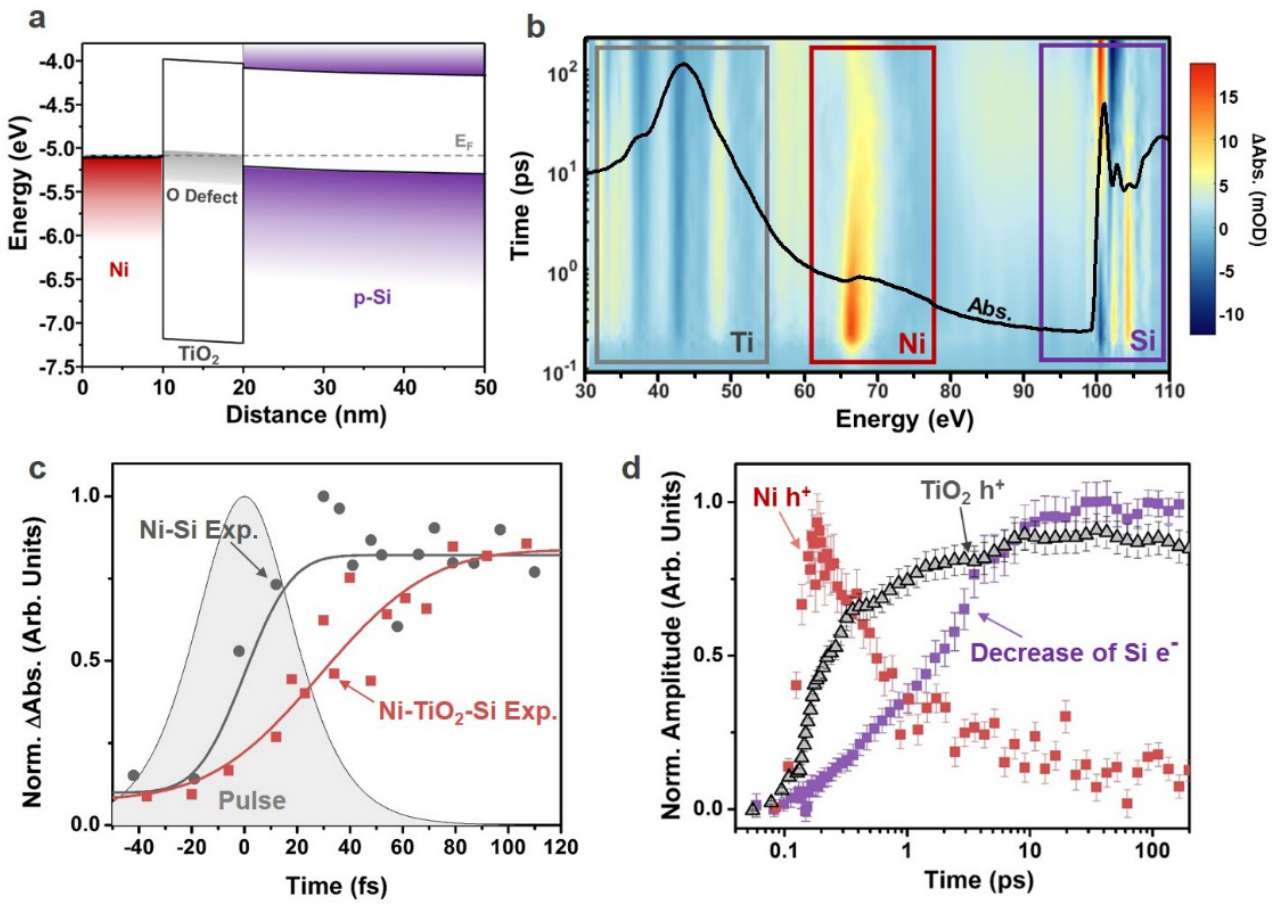

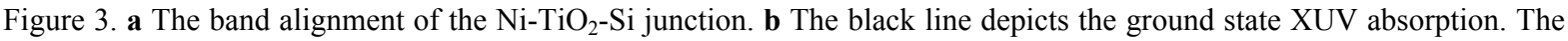
Ti $\mathrm{M}_{2,3}$, the $\mathrm{Ni} \mathrm{M}_{2,3}$, and the $\mathrm{Si} \mathrm{L}_{2,3}$ edges are energetically separated and feature unique time-dependent responses following the optical photoexcitation of Si. $\mathbf{c}$ The short time dynamics at the Ni edge indicate a hole tunneling that is prolonged in the presence of the $\mathrm{TiO}_{2}$ layer. The pulse is shown as the grey area. $\mathbf{d}$ The kinetics of the electrons and holes measured for each element of the junction on a logarithmic scale from $50 \mathrm{fs}$ to $200 \mathrm{ps}$ after photoexcitation. After the initial hole transfer, the $\mathrm{Ni}$ hole population decreases in correspondence with an increase in the hole population of the $\mathrm{TiO}_{2}$. The increased hole population on the $\mathrm{TiO}_{2}$ is then measured to correlate with a decrease in the photoexcited electron population on the $\mathrm{Si}$. Corresponding to this recombination, the hole population measured in the $\mathrm{TiO}_{2}$ decreases on a hundreds of picoseconds time scale. 


\subsection{Summary of core-hole perturbation effect on transient XUV spectra of solid state materials}

The core-hole excitation perturbs the final transition state, allowing charge- and heat-transfer processes to be measured from the XUV peak structure. The XUV spectrum analysis must consider the degree of localization of valence electrons, and thus the extent of the core-hole perturbation in the measured XUV spectrum (Figure 4). In materials with localized valence electrons, such as transition metal oxides, the core-hole perturbation is only weakly shielded by the excited state electrons. As a result, the excited state electron and hole energies are obscured by atomic multiplet splitting and charge transfer interactions. For such systems, transient XUV measurements are often limited to changes in oxidation states. In covalent semiconductors, the core-hole perturbation is shielded by delocalized $3 \mathrm{~s} / 3 \mathrm{p}$ electrons. Following an appropriate theoretical analysis, the photoexcited electron and hole energies can be extracted from the transient XUV spectrum. Furthermore, distortions of the lattice due to the averaged phonon modes can be quantified. In a metal, although core-hole shielding is largest, the XUV absorption features a broad spectrum that is not representative of the unoccupied density of states of the metal. The discrepancy is because the core-hole excitation results in an exponentially increasing number of possible electron configurations as the excitation energy approaches the Fermi level. The resultant many-body effect creates a strong white line edge of the absorption and a power law decaying tail. The exact line shape can be calculated as a function of the Fermi energy and temperature of the metal.
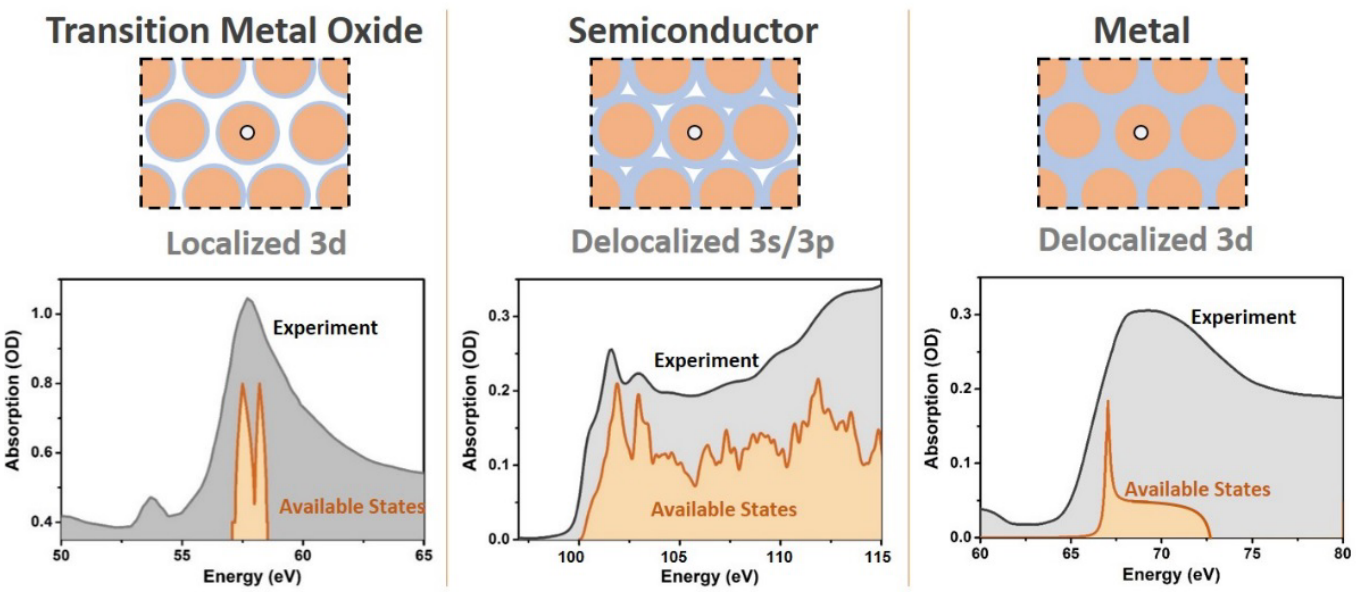

Figure 4. The XUV differential absorption in solid state materials follows the available density of states to varying degrees depending on the screening of the core hole interaction. In a metal oxide, the localized d levels interact strongly with the core-hole of the XUV transition. The final transition density of states is dominated by atomic multiple splitting and is therefore distorted from the unoccupied valence states. In a covalent semiconductor, the delocalized electrons shield the core-hole perturbation, and the measured experimental absorption is close to the unoccupied density of states. In metals, the white edge effect results in vanishing ground and excited state overlap at the Fermi level, resulting in large differential absorption relative to the available density of states.

\section{DETAILS OF TECHNIQUE}

High harmonic generation ( $\mathrm{HHG}$ ) enables few- to sub-femtosecond resolution with pulse durations that theoretically can reach tens of attoseconds ${ }^{29}$. In the semi-classical three-step model, the intense laser field causes electrons in the gas atoms to ionize via tunneling ${ }^{30}$. Upon sign change of the electric field, the ionized electrons are accelerated toward the atom and recombine, resulting in the emission of harmonic radiation. The energy cut-off for the HHG process is given by $h v_{\max }=I_{P}+3.16 U_{P}$, where $h$ is Planck's constant, $v_{\max }$ is the maximum frequency, $I_{P}$ is the ionization potential of the atomic gas, and $U_{P}$ is the ponderomotive energy, which is linearly proportional to the driving laser field intensity and the square of its wavelength. The cut-off energy therefore scales as $\lambda^{2}$ of the driving laser; however, the yield follows $\lambda^{-(5-6)}$, meaning higher harmonic energies are only now becoming feasible with the advent of more powerful infrared lasers and parametric amplifiers. 
XUV generation via HHG allows x-ray experiments to be conducted in a laboratory setting rather than in a synchrotron or free electron laser facility (FEL). In general, a few-cycle optical driving pulse is obtained by transmitting the visible or near-infrared (NIR) pulse through a differentially gas pumped hollow-core fiber, in which self-phase modulation with the carrier gas causes spectral broadening, followed by dispersion compensation. To compensate for group delay dispersion induced by transmissive optics, chirped mirrors are used for visible pulses. In the infrared, fused silica features anomalous dispersion thus making it suitable for temporally compressed NIR pulses. By tuning the dispersion compensation, visible and NIR pulses with time durations of $<5 \mathrm{fs}$ and $<12 \mathrm{fs}$, respectively, can be obtained $^{31,32}$. The high harmonics are generated by focusing the intense visible or NIR beam into a semi-infinite or infinite gas cell, which is flowed with a noble gas. Due to the energy scaling of high harmonic generation, the use of a NIR driving pulse allows access to higher energy $x$-ray edges in the water window ${ }^{8,29}$. A metal filter then removes residual visible or NIR light to select the desired harmonics. A portion of the beam used to create the high harmonics is usually used as the excitation beam, which is recombined with the XUV radiation on the sample after passing through an optomechanical time delay. Finally, the transmitted or reflected XUV radiation is filtered to remove the residual pump beam before being incident on an x-ray grating spectrometer equipped with a CCD camera.

Solid state transient XUV experiments have been implemented in both transmissive and reflective configuration, the latter of which offers increased flexibility in sample selection. Transient reflectivity directly measures the sample's full dielectric function $\varepsilon(\omega)=\varepsilon_{1}(\omega)+i \varepsilon_{2}(\omega)$, i.e. both its dispersive and absorptive components ${ }^{12,20}$. Additionally, transient reflectivity results in increased detection sensitivity at near-grazing angles and provides the opportunity to sample various depths due to the angle-dependence of $x$-ray penetration. The angle-dependent penetration depth has been used to map the spatio-chemical composition of layered nanomaterials ${ }^{33}$.

\section{CONCLUSIONS}

In conclusion, transient XUV spectroscopy features a rich, element-specific dataset that contains information about electronic and structural dynamics. The degree to which carrier and phonon kinetics can be measured depends on how the core-hole perturbation is screened. When an appropriate theoretical analysis is applied, transient XUV can measure the charge and heat transport within a nanoscale optoelectronic or photonic junction.

\section{ACKNOWLEDGEMENTS}

The represented and cited work was done by Scott Cushing and colleagues while in Stephen Leone's laboratory at the University of California, Berkeley. Scott Cushing was support by the Department of Energy, Office of Energy Efficiency and Renewable Energy (EERE) Postdoctoral Research Award under the EERE Solar Energy Technologies Office during this time. In writing this paper, Jonathan Michelsen was supported by the Institute Fellowship, California Institute of Technology.

\section{REFERENCES}

[1] Pop, E., "Energy dissipation and transport in nanoscale devices," Nano Res. 3(3), 147-169 (2010).

[2] J. Minnich, A., S. Dresselhaus, M., F. Ren, Z. and Chen, G., "Bulk nanostructured thermoelectric materials: current research and future prospects," Energy Environ. Sci. 2(5), 466-479 (2009).

[3] König, D., Casalenuovo, K., Takeda, Y., Conibeer, G., Guillemoles, J. F., Patterson, R., Huang, L. M. and Green, M. A., "Hot carrier solar cells: Principles, materials and design," Phys. E Low-Dimens. Syst. Nanostructures 42(10), 2862-2866 (2010).

[4] Shah, J., [Ultrafast Spectroscopy of Semiconductors and Semiconductor Nanostructures], Springer Berlin Heidelberg, Berlin, Heidelberg (1999).

[5] Shank, C. V. and Zakharchenia, B. P., [Spectroscopy of nonequilibrium electrons and phonons], NorthHolland, Amsterdam (1992).

[6] Reggiani, L., [Hot-Electron Transport in Semiconductors], Springer Berlin, Berlin (2014).

[7] Hentschel, M., Kienberger, R., Spielmann, C., Reider, G. A., Milosevic, N., Brabec, T., Corkum, P., Heinzmann, U., Drescher, M. and Krausz, F., “Attosecond metrology,” Nature 414, 509 (2001). 
[8] Johnson, A. S., Wood, D., Austin, D. R., Brahms, C., Gregory, A., Holzner, K. B., Jarosch, S., Larsen, E. W., Parker, S., Strüber, C., Ye, P., Tisch, J. W. G. and Marangos, J. P., "Apparatus for soft X-ray table-top high harmonic generation," Rev. Sci. Instrum. 89(8), 083110 (2018).

[9] Young, L., Ueda, K., Gühr, M., Bucksbaum, P. H., Simon, M., Mukamel, S., Rohringer, N., Prince, K. C., Masciovecchio, C., Meyer, M., Rudenko, A., Rolles, D., Bostedt, C., Fuchs, M., Reis, D. A., Santra, R., Kapteyn, H., Murnane, M., Ibrahim, H., et al., "Roadmap of ultrafast x-ray atomic and molecular physics," J. Phys. B At. Mol. Opt. Phys. 51(3), 032003 (2018).

[10] Kraus, P. M., Zürch, M., Cushing, S. K., Neumark, D. M. and Leone, S. R., "The ultrafast X-ray spectroscopic revolution in chemical dynamics," Nat. Rev. Chem. 2(6), 82-94 (2018).

[11] Kraus, P. M. and Wörner, H. J., "Perspectives of Attosecond Spectroscopy for the Understanding of Fundamental Electron Correlations," Angew. Chem. Int. Ed. 57(19), 5228-5247 (2018).

[12] Kaplan, C. J., Kraus, P. M., Ross, A. D., Zürch, M., Cushing, S. K., Jager, M. F., Chang, H.-T., Gullikson, E. M., Neumark, D. M. and Leone, S. R., "Femtosecond tracking of carrier relaxation in germanium with extreme ultraviolet transient reflectivity," Phys. Rev. B 97(20) (2018).

[13] Zürch, M., Chang, H.-T., Borja, L. J., Kraus, P. M., Cushing, S. K., Gandman, A., Kaplan, C. J., Oh, M. H., Prell, J. S., Prendergast, D., Pemmaraju, C. D., Neumark, D. M. and Leone, S. R., "Direct and simultaneous observation of ultrafast electron and hole dynamics in germanium," Nat. Commun. 8, 15734 (2017).

[14] Zürch, M., Chang, H.-T., Kraus, P. M., Cushing, S. K., Borja, L. J., Gandman, A., Kaplan, C. J., Oh, M. H., Prell, J. S., Prendergast, D., Pemmaraju, C. D., Neumark, D. M. and Leone, S. R., "Ultrafast carrier thermalization and trapping in silicon-germanium alloy probed by extreme ultraviolet transient absorption spectroscopy," Struct. Dyn. 4(4), 044029 (2017).

[15] Cushing, S. K., Zürch, M., Kraus, P. M., Carneiro, L. M., Lee, A., Chang, H.-T., Kaplan, C. J. and Leone, S. R., "Hot phonon and carrier relaxation in $\mathrm{Si}(100)$ determined by transient extreme ultraviolet spectroscopy," Struct. Dyn. 5(5), 054302 (2018).

[16] Carneiro, L. M., Cushing, S. K., Liu, C., Su, Y., Yang, P., Alivisatos, A. P. and Leone, S. R., "Excitation-wavelength-dependent small polaron trapping of photoexcited carriers in $\alpha$-Fe2O3," Nat. Mater. 16(8), 819-825 (2017).

[17] Buzzi, M., Först, M., Mankowsky, R. and Cavalleri, A., "Probing dynamics in quantum materials with femtosecond X-rays," Nat. Rev. Mater. 3(9), 299-311 (2018).

[18] Baker, L. R., Jiang, C.-M., Kelly, S. T., Lucas, J. M., Vura-Weis, J., Gilles, M. K., Alivisatos, A. P. and Leone, S. R., "Charge Carrier Dynamics of Photoexcited Co3O4 in Methanol: Extending High Harmonic Transient Absorption Spectroscopy to Liquid Environments," Nano Lett. 14(10), 5883-5890 (2014).

[19] Gilbert, B., Frandsen, C., Maxey, E. R. and Sherman, D. M., "Band-gap measurements of bulk and nanoscale hematite by soft x-ray spectroscopy," Phys. Rev. B 79(3) (2009).

[20] Biswas, S., Husek, J., Londo, S. and Baker, L. R., "Ultrafast Electron Trapping and Defect-Mediated Recombination in NiO Probed by Femtosecond Extreme Ultraviolet Reflection-Absorption Spectroscopy," J. Phys. Chem. Lett. 9(17), 5047-5054 (2018).

[21] Vura-Weis, J., Jiang, C.-M., Liu, C., Gao, H., Lucas, J. M., de Groot, F. M. F., Yang, P., Alivisatos, A. P. and Leone, S. R., "Femtosecond M2,3-Edge Spectroscopy of Transition-Metal Oxides: Photoinduced Oxidation State Change in $\alpha$-Fe2O3," J. Phys. Chem. Lett. 4(21), 3667-3671 (2013).

[22] Husek, J., Cirri, A., Biswas, S. and Baker, L. R., "Surface electron dynamics in hematite $\left(\alpha-\mathrm{Fe}_{2} \mathrm{O}_{3}\right)$ : correlation between ultrafast surface electron trapping and small polaron formation," Chem. Sci. 8(12), 8170-8178 (2017).

[23] Porter, I. J., Cushing, S. K., Carneiro, L. M., Lee, A., Ondry, J. C., Dahl, J. C., Chang, H.-T., Alivisatos, A. P. and Leone, S. R., "Photoexcited Small Polaron Formation in Goethite $(\alpha-\mathrm{FeOOH})$ Nanorods Probed by Transient Extreme Ultraviolet Spectroscopy,” J. Phys. Chem. Lett. 9(14), 4120-4124 (2018). 
[24] Biswas, S., Husek, J., Londo, S., Fugate, E. A. and Baker, L. R., "Identifying the acceptor state in NiO hole collection layers: direct observation of exciton dissociation and interfacial hole transfer across a $\mathrm{Fe}$ ${ }_{2} \mathrm{O}_{3} / \mathrm{NiO}$ heterojunction," Phys. Chem. Chem. Phys. 20(38), 24545-24552 (2018).

[25] Kaplan, C. J., Kraus, P. M., Ross, A. D., Zürch, M., Cushing, S. K., Jager, M. F., Chang, H.-T., Gullikson, E. M., Neumark, D. M. and Leone, S. R., "Femtosecond tracking of carrier relaxation in germanium with extreme ultraviolet transient reflectivity," Phys. Rev. B 97(20) (2018).

[26] Richter, A., Glunz, S. W., Werner, F., Schmidt, J. and Cuevas, A., "Improved quantitative description of Auger recombination in crystalline silicon," Phys. Rev. B 86(16), 165202 (2012).

[27] Ohtaka, K. and Tanabe, Y., "Golden-rule approach to the soft-x-ray-absorption problem. III. The temperature dependence," Phys. Rev. B 30(8), 4235-4258 (1984).

[28] Ohtaka, K. and Tanabe, Y., "Theory of the soft-x-ray edge problem in simple metals: historical survey and recent developments," Rev. Mod. Phys. 62(4), 929-991 (1990).

[29] Popmintchev, T., Chen, M.-C., Popmintchev, D., Arpin, P., Brown, S., Alisauskas, S., Andriukaitis, G., Balciunas, T., Mucke, O. D., Pugzlys, A., Baltuska, A., Shim, B., Schrauth, S. E., Gaeta, A., HernandezGarcia, C., Plaja, L., Becker, A., Jaron-Becker, A., Murnane, M. M., et al., "Bright Coherent Ultrahigh Harmonics in the keV X-ray Regime from Mid-Infrared Femtosecond Lasers," Science 336(6086), 1287-1291 (2012).

[30] Lewenstein, M., Balcou, P., Ivanov, M. Y., L'Huillier, A. and Corkum, P. B., "Theory of high-harmonic generation by low-frequency laser fields," Phys. Rev. A 49(3), 2117-2132 (1994).

[31] Nisoli, M., De Silvestri, S., Svelto, O., Szipöcs, R., Ferencz, K., Spielmann, C., Sartania, S. and Krausz, F., "Compression of high-energy laser pulses below 5 fs," Opt. Lett. 22(8), 522 (1997).

[32] Brida, D., Cirmi, G., Manzoni, C., Bonora, S., Villoresi, P., Silvestri, S. D. and Cerullo, G., "Sub-twocycle light pulses at $1.6 \mu \mathrm{m}$ from an optical parametric amplifier," Opt. Lett. 33(7), 741-743 (2008).

[33] Majhi, A., Nayak, M., Pradhan, P. C., Filatova, E. O., Sokolov, A. and Schäfers, F., "Soft X-ray Reflection Spectroscopy for Nano-Scaled Layered Structure Materials," Sci. Rep. 8(1) (2018). 